\title{
Radiation Therapy Is a Reasonable Option for Improving the Prognosis in Hepatocellular Carcinoma
}

\author{
Yasuteru Kondo, ${ }^{1, *}$ Osamu Kimura, ${ }^{1, *}$ Takayuki Kogure, ${ }^{1}$ Masashi Ninomiya, ${ }^{1}$ \\ Rei Umezawa, ${ }^{2}$ Toshiyuki Sugawara, ${ }^{2}$ Haruo Matsushita, ${ }^{2}$ Keiichi Jingu, ${ }^{2}$ \\ Yu Nakagome, ${ }^{1}$ Tomoaki Iwata, ${ }^{1}$ Tatsuki Morosawa, ${ }^{1}$ Yasuyuki Fujisaka, ${ }^{1}$ \\ Takao Iwasaki ${ }^{1}$ and Tooru Shimosegawa ${ }^{1}$
}

${ }^{1}$ Division of Gastroenterology, Tohoku University Hospital, Sendai, Miyagi, Japan

${ }^{2}$ Division of Radiation Oncology, Tohoku University Hospital, Sendai, Miyagi, Japan

\begin{abstract}
Radiation therapy (RT) may be suitable for treating patients with hepatocellular carcinoma (HCC) who are difficult to treat with any other option. However, it remains unclear whether RT extends survival in these patients. Among the $957 \mathrm{HCC}$ patients treated at Tohoku University Hospital from January 2007 to December 2013, only 49 patients received RT. We therefore retrospectively analyzed the outcomes of these patients; they were divided into three groups based on the reasons for choosing RT: 27 patients at Stage IV A $(67.1 \pm 1.6$ years, $50.5 \pm 2.1 \mathrm{~Gy}), 9$ patients with alternative therapy $(72.2 \pm 2.4$ years, $58.9 \pm 1.1$ Gy), and 13 patients who received RT after transarterial chemoembolization (TACE) (75.6 \pm 2.1 years, 56.5 $\pm 1.5 \mathrm{~Gy}$ ). RT was employed to ensure the local control of the lesion. The patients at Stage IV A were treated with radical $R T(n=16)$ or with palliative $R T(n=11)$. In radical RT group, the response rate was $37.5 \%$ and the complete response rate was $25 \%$. The survival rate was $12.5 \pm 2.6$ months after radical RT. This is considered relatively good for Stage IV A. The disease-free survival rate was $13.0 \pm 2.8$ months after RT. This excellent disease-free survival indicates that RT is an alternative to other treatments. In the TACE group, patients who received the RT had the significantly long disease-free survival rate than onlyTACE $(18.0 \pm 3.8$ months vs. $11.2 \pm 0.58$ months $)$. We propose that RT is effective and safe for HCC.
\end{abstract}

Keywords: hepatocellular carcinoma; portal vein thrombosis; radiation therapy; radio-frequency ablation; transarterial chemoembolization

Tohoku J. Exp. Med., 2015 December, 237 (4), 249-257. C 2015 Tohoku University Medical Press

\section{Introduction}

Worldwide, the mortality from hepatocellular carcinoma (HCC) represents one-third of all cancer-related deaths, with more than 1 million per year (Lau and Lai 2008). Viral hepatitis due to hepatitis $\mathrm{C}$ or B virus (HCV or HBV), and chronic liver diseases such as alcoholic and non-alcoholic steatohepatitis (NASH) are critical factors in the development of HCC. Among these causes, chronic infection with $\mathrm{HCV}$ or $\mathrm{HBV}$ can strongly affect the incidence of HCC (Mittal and El-Serag 2013). The age of HCC patients is affected by the cause of HCC. The age of HCC patients in Japan is higher than in other Asian-Pacific countries due to the incidence of HCV-related HCC (Kim et al. 2013).

In the early stages of $\mathrm{HCC}$, when patients maintain a hepatic functional reserve, local treatment such as hepatic resection or radio-frequency ablation (RFA) is effective to control the progression of HCC. However, hepatic resection may become difficult for patients with poor hepatic function, and RFA may become difficult for those with multiple HCC lesions or vessel invasion with repeated recurrences. For patients not suitable for resection or RFA, transarterial chemoembolization (TACE) is one of the most significant treatment options since TACE can be carried out repeatedly. Moreover, TACE treatment is possible in patients with low hepatic functional reserve or with advanced HCC. The curative effect of TACE is controversial, but some randomized trials have shown improved survival using TACE (Lo et al. 2002; Llovet et al. 2002).

Sorafenib, an oral multikinase inhibitor, has recently been used for advanced HCC patients. Sorafenib was shown to be effective in two randomized studies (Llovet et al. 2008; Cheng et al. 2009). However, satisfactory results have not yet been shown.

Recently, various types of radiation therapy (RT) such

Received March 12, 2015; revised and accepted October 9, 2015. Published online November 12, 2015; doi: 10.1620/tjem.237.249.

*These two authors contributed equally to this work.

Correspondence: Yasuteru Kondo, Division of Gastroenterology, Tohoku University Hospital, 1-1 Seiryo-machi, Aoba-ku, Sendai, Miyagi 980-8574, Japan.

e-mail: yasuteru@ebony.plala.or.jp 
as stereotactic radiotherapy (Lo et al. 2010) and particle radiotherapy have been developed, and good treatment results have been reported (Komatsu et al. 2011). The number of patients treated with RT for HCC has been increasing rapidly. However, its effectiveness is still unclear. Each study used a different method of RT and a different total irradiation dose (Feng and Ben-Josef 2011).

Currently, RT is not described in the Barcelona Clinic Liver Cancer system (Llovet et al. 1999; Klein and Dawson 2013) or in the treatment algorithm by the Japan Society of Hepatology (Kudo et al. 2011). However, the tolerability of RT may be better than in other local treatments for HCC, especially in elderly patients. Accordingly, we have analyzed the RT cases conducted in our hospital retrospectively to confirm its effect.

\section{Materials and Methods}

\section{Patients}

Nine hundred and fifty-seven HCC patients were treated in our hospital from January 2007 to December 2013, including patients who received several rounds of treatment. Forty-nine patients received RT for intrahepatic HCC (see Fig. 1A). The diagnosis of $\mathrm{HCC}$ was based on the characteristic image findings from ultrasound, computed tomography (CT), magnetic resonance imaging (MRI), and serum tumor markers, $\alpha$-fetoprotein and PIVKA-II (protein induced by vitamin $\mathrm{K}$ absence or antagonist-II). Patients with extrahepatic metastases of HCC were excluded. We reviewed the reason, patient background, treatment effect, and survival rate for the selected RT. This study was approved by the Ethics Committee of Tohoku University School of Medicine (2014-1-434).

\section{Radiation therapy}

RT planning was performed using ECLIPSE (Varian Medical Systems, Palo Alto, CA). RT was delivered using coplanar and/or noncoplanar beams of 10-megavoltage equipment with a multileaf collimator. All patients were treated with a Clinac iX Linear Accelerator (Varian Medical Systems, Palo Alto, CA). The gross tumor volume (GTV) was defined as the contrast-enhancing lesions on the early arterial phase and/or portal vein thrombosis. The clinical target volume (CTV) was defined as the GTV plus a 5-10-mm margin. The planning target volume (PTV) was defined as the CTV plus a 5-10-mm margin. The cranial and caudal margin was about 10-20 $\mathrm{mm}$ with consideration for liver motion due to respiration movement. With some patients, abdominal compression was used to reduce respiration movement. RT was administered in fractions of 1.8 to 5.0 Gy daily $(2.4 \pm 0.67)$, five times a week. The total dose ranged from 12 Gy to 60 Gy $(53.5 \pm 9.5)$. The total dose was determined by radiation oncologists, based on age, performance status, liver function, and dose volume of the normal liver, duodenum, and stomach. Radical RT intended for patients who could be irradiated to the entire lesion. On the other hand, palliative RT intended for the patient who irradiated a part of tumor for the control of the tumor thrombus.

\section{Transarterial chemoembolization}

Digital subtraction angiography (DSA) was performed in all patients before TACE. The Seldinger technique was used to perform TACE in our hospital. DSA of the superior mesenteric artery and celiac trunk was performed to detect the HCC and its feeding artery.
A mixture of cisplatin (30-40 mg), doxorubicin hydrochloride (30-40 $\mathrm{mg}$ ), mitomycin C (6-8 mg), and nonionic soluble contrast media (80 $\mathrm{ml}$ ) was injected slowly from the proper hepatic artery or the first branch of the hepatic artery. The tip of the catheter was introduced into the feeding artery and used to inject iodized oil and gelfoam for embolization. In the TACE plus irradiation group, RT was started 4 to 8 weeks after TACE.

\section{Evaluation and analysis}

Patients were monitored with blood tests and CT depending on their condition, and were examined for the treatment effect of the RT after six months to detect any recurrence. We evaluated the curative effect using the Response Evaluation Criteria in Solid Tumors (RECIST) (Therasse et al. 2000). Complete disappearance of the tumor was considered as a complete response (CR), a decrease in the tumor size $\geq 50 \%$ as a partial response (PR), a decrease in the tumor $<50 \%$ or no change as stable disease (SD), and progression as progressive disease (PD). The objective response rate was calculated for the proportion of patients with a reduction in tumor size (CR and PR). Disease-free survival was estimated from the end of RT according to the Kaplan-Meier method. Statistical analyses were performed using JMP pro version 9.0 software. The data were evaluated with MannWhitney's $U$ test or a $\chi^{2}$ test. The data were also evaluated with a logrank test and a Wilcoxon test. Values of $p<0.05$ were defined as statistically significant.

\section{Results \\ Indications to select radiation therapy}

Patients who received RT were divided into three groups according to their symptomatic state (Fig. 1A, Table 1). The first group of patients consisted of those who were not indicated for hepatic resection and other local treatments because of vascular invasion: the Stage IV A group (n $=27$ ). The second group of patients was the alternative therapy group $(n=9)$. This group had a lesion for which local treatment was indicated (surgery, RFA, or TACE) but they selected RT as a substitute because there was a difficulty in proceeding with local treatment. The third group comprised patients who received RT after TACE to ensure the local control of the lesion: the TACE group $(n=13)$. The Stage IV A group was younger, had poorer liver function, and received a lower RT dose in comparison with the other groups (Table 1).

The histories of previous treatments in the three groups are shown in Fig. 1B. Pretreatments were given to the Stage IV A group for 64 times, the alternative therapy group for 20 times, and the TACE group for 32 times, respectively. The total number of previous treatments was highest in the Stage IV A group.

\section{Stage IV A group}

Table 2 shows the clinical profiles of the Stage IV A patients $(\mathrm{n}=27)$. The total RT dose was $50.5 \pm 11.2 \mathrm{~Gy}$ in this group (radical RT was $54.8 \pm 5.0 \mathrm{~Gy}$ and palliative RT was $44.4 \pm 14.7 \mathrm{~Gy}$ ). Treatment for this patient group was originally planned to be hepatic arterial infusion chemotherapy (HAIC) or sorafenib. However, these treatments 

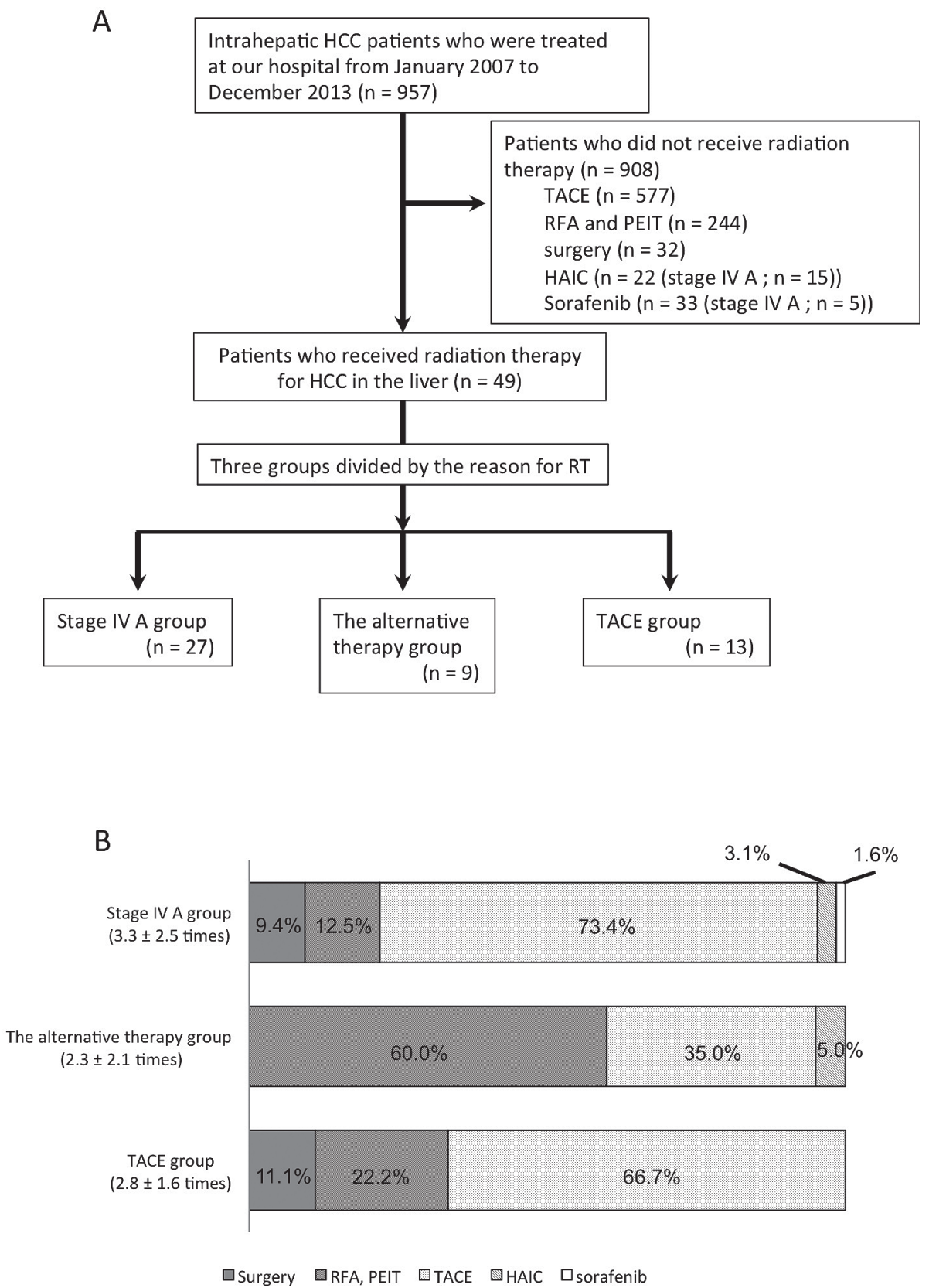

Fig. 1. Schematic design of the enrollment of the RT.

(A) Flow chart of the patients who received radiation therapy in this study. The 908 patients did not receive radiation therapy. The 577 patients received TACE, including the 120 patients who received TACE for the first time. The 244 patients received RFA and percutaneous ethanol injection therapy (PEIT) and the 32 patients received surgery. From these 276 patients, the 46 patients were selected as control of TACE group (RT+TACE). (B) The histories of previous treatments in the three groups are shown. The number of treatments is shown in parentheses (mean \pm SD). Pretreatments were given to the patients of the Stage IV A group for 64 times, the patients undergoing the alternative therapy for 20 times, and the patients with TACE for 32 times, respectively. The number in the box shows a ratio of indicated treatment to the number of the overall treatments in each group.

were abandoned because of the localization of the HCC, the liver function reserve, complications, older age, or difficulty in isolating the blood supply because of extra hepatic vessels. This group showed high levels of tumor markers, numerous tumors, and large tumors. As for the portal vein thrombosis, 17 cases were $<\mathrm{Vp} 3$ and 10 cases were $\geq \mathrm{Vp} 3$.

The Stage IV A group was divided into the radical irra- diation group $(n=16)$ and the palliative irradiation group ( $\mathrm{n}$ $=11$ ). In the radical RT group, CR was observed in 4 patients $(25 \%)$. The response rate was $37.5 \%(\mathrm{CR}, \mathrm{n}=4$; $\mathrm{PR}, \mathrm{n}=2 ; \mathrm{SD}, \mathrm{n}=1$; and $\mathrm{PD}=9$ ). There were no cases of treatment discontinuation owing to side effects. The median survival period after RT was $12.5 \pm 2.6$ months (Fig. 2A). Eleven patients were treated with palliative RT. 
Table 1. Clinical profiles of the three groups that received radiation therapy.

\begin{tabular}{|c|c|c|c|c|}
\hline & $\begin{array}{l}\text { Stage IV A group } \\
\qquad(\mathrm{n}=27)\end{array}$ & $\begin{array}{l}\text { The alternative } \\
\text { therapy group } \\
(\mathrm{n}=9)\end{array}$ & $\begin{array}{l}\text { TACE group } \\
(\mathrm{n}=13)\end{array}$ & \\
\hline Age & $67.1 \pm 1.6$ & $72.2 \pm 2.4$ & $75.8 \pm 2.1$ & $p=0.0072$ \\
\hline Sex (male : female) & $21: 6$ & $5: 4$ & $4: 9$ & $p=0.0156$ \\
\hline Child-Pugh (A : B : C) & $16: 11: 0$ & $9: 0: 0$ & $13: 0: 0$ & $p=0.0031$ \\
\hline Etiology (HCV : HBV : NBNC) & $16: 4: 7$ & $6: 0: 3$ & $9: 1: 3$ & $p=0.7471$ \\
\hline RT dose (Gy) & $50.5 \pm 2.1$ & $58.9 \pm 1.1$ & $56.5 \pm 1.5$ & $p=0.013$ \\
\hline
\end{tabular}

Data are expressed as mean \pm SE. The RT dose was analyzed by Wilcoxon's test. Other data were analyzed by the $\chi^{2}$ test.

Table 2. Clinical profiles of the Stage IV A group.

\begin{tabular}{|c|c|c|c|}
\hline & $\begin{array}{l}\text { Radical RT } \\
(\mathrm{n}=16)\end{array}$ & $\begin{array}{l}\text { Palliative RT } \\
\quad(\mathrm{n}=11)\end{array}$ & $\begin{array}{c}\text { All } \\
(n=27)\end{array}$ \\
\hline Age & $66.6 \pm 2.4$ & $67.1 \pm 1.6$ & $67.1 \pm 1.6$ \\
\hline Sex (male : female) & $13: 3$ & $8: 3$ & $21: 6$ \\
\hline Child-Pugh (A : B : C) & $11: 5: 0$ & $5: 6: 0$ & $16: 11: 0$ \\
\hline Etiology (HCV : HBV : NBNC) & $10: 0: 6$ & $6: 4: 1$ & $16: 4: 7$ \\
\hline AFP & $3,463 \pm 3,177$ & $53,899 \pm 46,842$ & $24,918 \pm 19,992$ \\
\hline AFP-L3 & $28.5 \pm 6.5$ & $45.6 \pm 8.0$ & $35.7 \pm 5.4$ \\
\hline PIVKA-II & $688.4 \pm 276.5$ & $3,646 \pm 2,209$ & $2,072 \pm 1,043$ \\
\hline Tumor size $(\mathrm{cm})$ & $4.5 \pm 0.4$ & $5.9 \pm 1.0$ & $5.1 \pm 0.5$ \\
\hline Tumor number $(<4: \geq 4)$ & $4: 12$ & $3: 8$ & $7: 20$ \\
\hline TNM staging (I : II : III : IV A) & $0: 0: 0: 16$ & $0: 0: 0: 11$ & $0: 0: 0: 27$ \\
\hline CLIP score $(0: 1: 2: 3: 4: 5)$ & $0: 1: 4: 6: 3: 2$ & $0: 0: 1: 2: 8: 0$ & $0: 1: 5: 8: 11: 2$ \\
\hline Portal vein thrombosis $(<\mathrm{Vp} 3: \geq \mathrm{Vp} 3)$ & $12: 4$ & $5: 6$ & $17: 10$ \\
\hline Radical irradiation:palliative irradiation & & & $16: 11$ \\
\hline
\end{tabular}

Data are expressed as mean \pm SE. AFP, alpha-fetoprotein; AFP-L3 (\%), LCA-reactive alpha-fetoprotein isoform; PIVKAII, protein induced by vitamin K absence or antagonists-II. TNM staging (the Liver Cancer Study Group of Japan), CLIP score (Cancer of the Liver Italian Program).

In palliative RT, seven patients received RT to control the tumor growth and portal vein thrombosis. Three patients showed improvement in obstructive jaundice, and hemostasis of biliary tract bleeding was present in one patient. Two patients discontinued treatment because of cytopenia and liver abscesses, respectively. In the palliative RT group, the median survival period after RT was $5.5 \pm 1.0$ months. The median survival period after RT was $9.7 \pm 2.6$ months in the Stage IV A group.

The radical irradiation group was compared with the sorafenib group with Stage IV A disease (Table 3). A trend towards longer survival was observed in the RT group (Fig. 2B).

\section{The alternative therapy group}

Table 4 shows the clinical profiles of the alternative therapy patients $(\mathrm{n}=9)$. The total RT dose was $58.9 \pm 3.3$ Gy for this group. All patients of this group had maintained their liver reserve; however, it was difficult to proceed with local treatment owing to other on-going treatments for dementia, dissecting aneurysm, or extrahepatic blood ves- sels that had a risk for embolus. In the alternative therapy group, a CR was observed in three patients $(33 \%)$. The response rate was $56 \%(\mathrm{CR}, \mathrm{n}=3 ; \mathrm{PR}, \mathrm{n}=2 ; \mathrm{SD}, \mathrm{n}=2$; and $\mathrm{PD}, \mathrm{n}=2$ ). The median disease-free survival period after RT was $13.0 \pm 2.8$ months, and the median overall survival rate after RT was $29.2 \pm 3.6$ months (Fig. 3A). RT was not the first treatment for these patients. Therefore, this group had a comparatively long overall survival rate of $101.9 \pm 17.9$ months (Fig. 3B).

\section{TACE group}

RT was added to TACE to further enhance the effect of treatment with TACE in this group. The total RT dose was 56.3.9 $\pm 5.7 \mathrm{~Gy}$. The patients in this group did not undergo surgery or RFA, because of the difficulty in performing RFA or surgery due to the location of the HCC, aging and/ or impaired liver function. However, they received RFA or surgery in the previous treatment (see Fig. 1B). In some cases, the patient's preference dictated the choice of treatment. Many of these patients maintained their liver reserve functions but could not tolerate surgery because of their 
Table 3. Clinical profiles of the Stage IV A (radical irradiation) group and patients who received sorafenib.

\begin{tabular}{lccc}
\hline & $\begin{array}{c}\text { RT (radical irradiation) } \\
(\mathrm{n}=16)\end{array}$ & $\begin{array}{c}\text { Sorafenib } \\
(\mathrm{n}=5)\end{array}$ \\
\hline Age & $66.6 \pm 2.4$ & $60.2 \pm 1.1$ & $p=0.1593$ \\
Sex (male $:$ female) & $13: 3$ & $5: 0$ & $p=0.1819$ \\
Child-Pugh (A : B : C) & $11: 5: 0$ & $2: 3: 0$ & $p=0.2479$ \\
Etiology (HCV : HBV : NBNC) & $10: 0: 6$ & $3: 2: 0$ & $p=0.0159 *$ \\
AFP & $3,463 \pm 3,177$ & $4,452 \pm 4,432$ & $p=0.4326$ \\
AFP-L3 & $28.5 \pm 6.5$ & $18.0 \pm 12.1$ & $p=0.4521$ \\
PIVKA-II & $688.4 \pm 276.5$ & $6,532.6 \pm 5,234.2$ & $p=0.5786$ \\
Tumor size (cm) & $4.5 \pm 0.4$ & $5.5 \pm 1.5$ & $p=0.7408$ \\
Tumor number $(<4: \geq 4)$ & $4: 12$ & $0: 3: 0: 4: 1: 0$ & $p=0.5169$ \\
CLIP score $(0: 1: 2: 3: 4: 5)$ & $0: 1: 4: 6: 3: 2$ & $3: 1: 1$ & $p=0.4576$ \\
Portal vein thrombosis (non $:<$ Vp3 $: \geq$ Vp3) & $0: 12: 4$ & $p=0.1593$ \\
\hline
\end{tabular}

Data are expressed as mean \pm SE. Age, AFP, AFP-L3, PIVKA-II, Tumor sizes were analyzed by Wilcoxon's test. Other data were analyzed by the $\chi^{2}$ test.

$* p<0.05$.

A

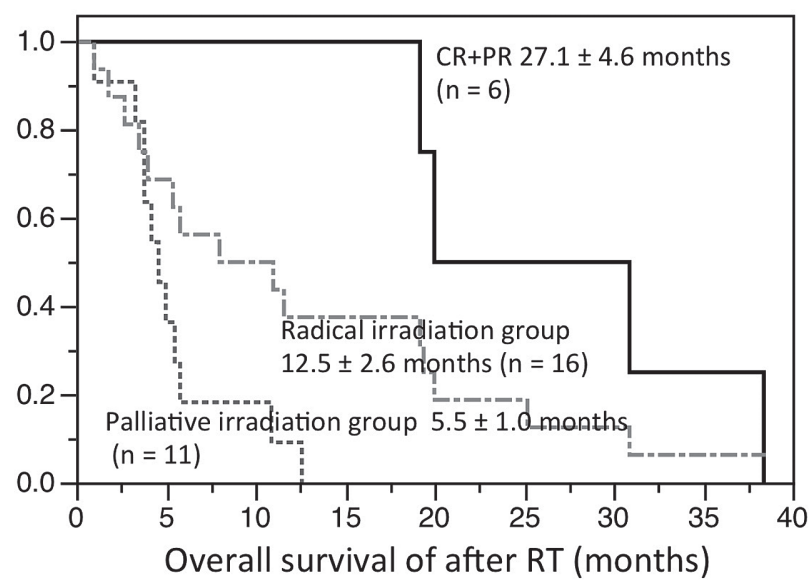

B

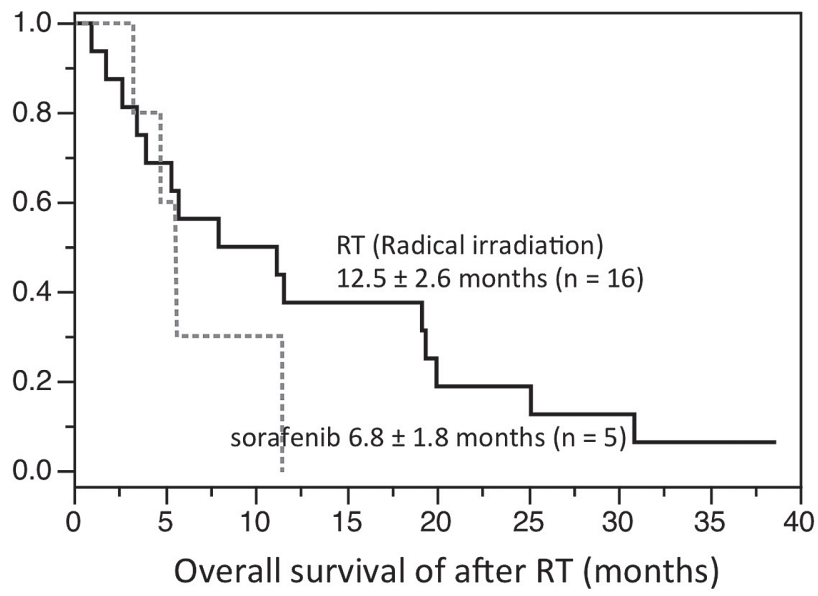

Fig. 2. Overall survival rate of after RT in Stage IV A group. (A) The 6 patients among the 16 patients with radical irradiation becomes $\mathrm{CR}$ or $\mathrm{PR}(\mathrm{CR}, \mathrm{n}=4 ; \mathrm{PR}, \mathrm{n}=2)$. Overall response rate (CR and PR) were shown. (B) Overall survival of the radical irradiation group (RT) and the sorafenib group.
A

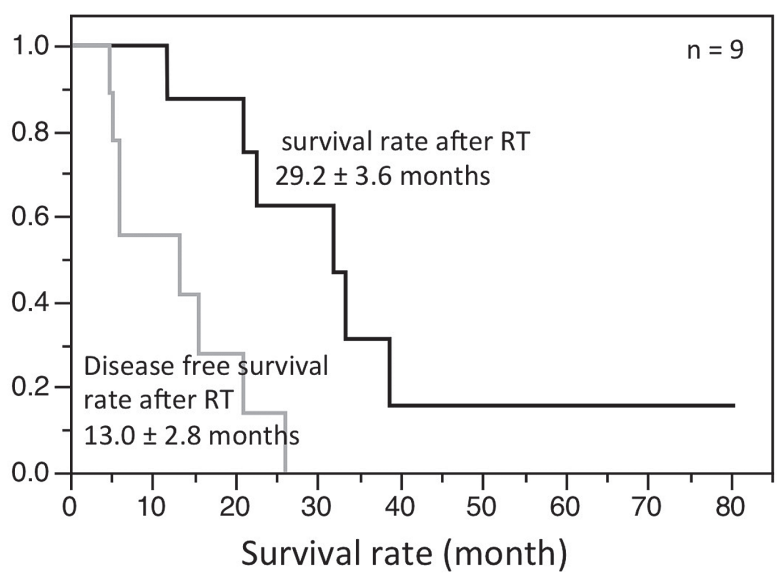

B

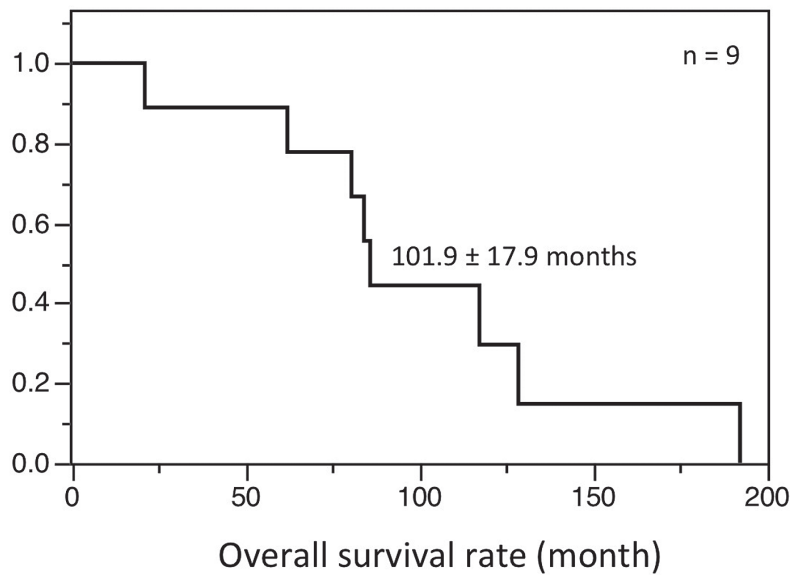

Fig. 3. Survival rate of after RT in the alternative therapy group.

(A) Disease-free survival rate and survival rate after RT in the alternative therapy group. (B) Counting the first treatment of the alternative therapy group as time zero, this shows the overall survival rate. 
age-related low cardiopulmonary function. Therefore, this group was the oldest among the three groups. One Stage IV A patient was included in this group. This patient was less than Vp3, and TACE was carried out for the tumor thrombus. In order to enhance the therapeutic effect, RT was also administered. Therefore, we added this patient to the TACE group.

In the TACE group, $\mathrm{CR}$ was observed in eight patients (62\%). The response rate was $69.2 \%$ (CR 8, PR 1, SD 1, and PD 3). After one attempt, the TACE group was compared to first HCC treatment (RFA or surgery) patients (Table 5). Among 907 patients treated HCC at our hospital between January 2006 to December 2012, we chose 276 patients who received local treatment (RFA or PEIT) or resection. Finally, among the 276 patients, we chose 46 patients of more than $10 \mathrm{~mm}$ tumor diameter without HCC treatment history as a historical control (Fig. 1A, Table 5). Patients with lesions of $1>\mathrm{cm}$ and multiple lesions were excluded.

The median disease-free survival period was $18.0 \pm 3.8$

Table 4. Clinical profiles of the alternative therapy group.

\begin{tabular}{lc}
\hline & $\mathrm{n}=9$ \\
\hline Age & $72.2 \pm 2.4$ \\
Sex (male $:$ female) & $5: 4$ \\
Child-Pugh $(\mathrm{A}: \mathrm{B}: \mathrm{C})$ & $9: 0: 0$ \\
Etiology $(\mathrm{HCV}: \mathrm{HBV}:$ NBNC) & $6: 0: 3$ \\
AFP & $190.3 \pm 174.1$ \\
AFP-L3 & $11.4 \pm 9.7$ \\
PIVKA-II & $490.3 \pm 352.0$ \\
Tumor size $(\mathrm{cm})$ & $2.7 \pm 0.3$ \\
Tumor number $(1: 2-3: \geq 4)$ & $6: 1: 2$ \\
TNM staging $(\mathrm{I}: \mathrm{II}: \mathrm{III}:$ IV A) & $3: 3: 3: 0$ \\
CLIP score $(0: 1: 2: 3: 4: 5)$ & $5: 3: 1: 0: 0: 0$ \\
\hline
\end{tabular}

Data expressed as mean $\pm \mathrm{SE}$.
A

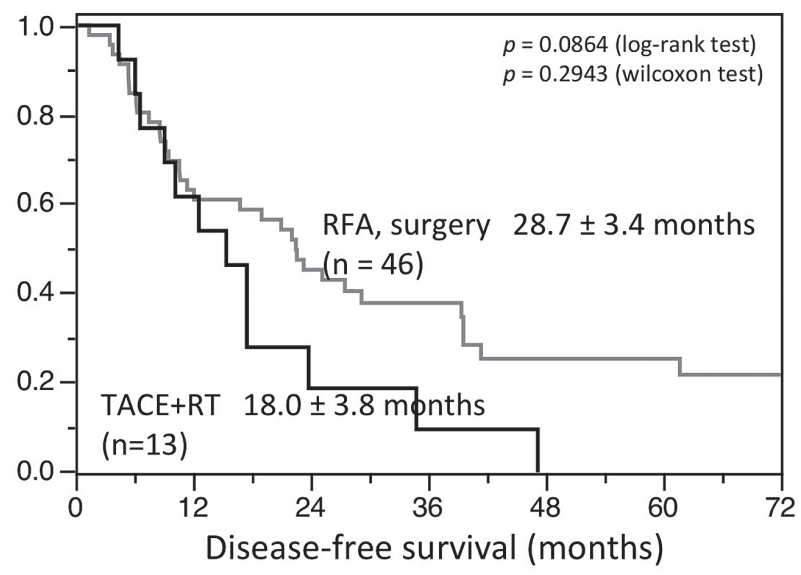

B

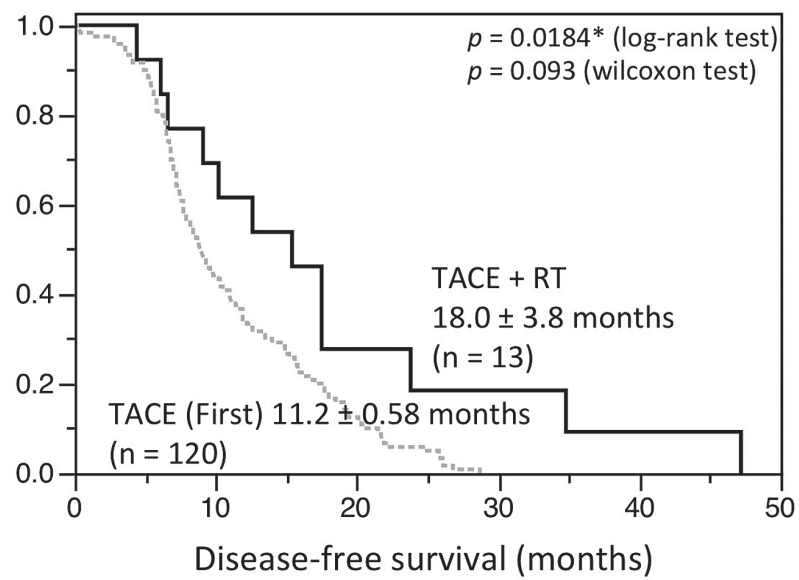

Fig. 4. Survival rate of after RT in the TACE group. (A) Disease-free survival rate after RT in the TACE group and the naïve HCC group (RFA or surgery). (B) Disease-free survival rate after RT in the TACE group and first TACE group. The data were evaluated with a log-rank test and a Wilcoxon test.

Table 5. Clinical profiles of the TACE group and the control group (RFA or surgery).

\begin{tabular}{lccc}
\hline & $\begin{array}{c}\text { RFA or surgery } \\
(\mathrm{n}=46)\end{array}$ & $\begin{array}{c}\text { TACE }+ \text { RT } \\
(\mathrm{n}=13)\end{array}$ & \\
\hline Age & $66.5 \pm 1.4$ & $75.8 \pm 2.1$ & $p=0.0015$ \\
Sex (male $:$ female) & $29: 17$ & $4: 9$ & $p=0.0385$ \\
Child-Pugh (A : B : C) & $39: 7: 0$ & $13: 0: 0$ & $p=0.1341$ \\
Etiology (HCV : HBV : NBNC : HCV+HBV) & $31: 6: 7: 2$ & $9: 1: 3: 0$ & $p=0.7568$ \\
AFP & $129.3 \pm 61.4$ & $448.2 \pm 326.1$ & $p=0.4533$ \\
AFP-L3 & $5.9 \pm 1.7$ & $15.6 \pm 6.7$ & $p=0.3247$ \\
PIVKA-II & $424.5 \pm 216.3$ & $25.1 \pm 4.09$ & $p=0.0752$ \\
Tumor size (cm) & $2.3 \pm 0.3$ & $2.5 \pm 0.3$ & $p=0.1504$ \\
Tumor number $(1: 2-3: \geq 4)$ & $40: 6: 0$ & $11: 1: 1$ & $p=0.1092$ \\
TNM staging $(\mathrm{I}:$ II $:$ III $:$ IV A) & $24: 17: 5: 0$ & $5: 7: 0: 1$ & $p=0.1025$ \\
CLIP score $(0: 1: 2: 3: 4: 5)$ & $30: 15: 1: 0: 0$ & $5: 6: 1: 0: 1$ & $p=0.0015$ \\
\hline
\end{tabular}

Data are expressed as mean \pm SE. Age, AFP, AFP-L3, PIVKA-II, Tumor sizes were analyzed by Wilcoxon's test. Other data were analyzed by the $\chi^{2}$ test. 
Table 6. Clinical profiles of the TACE group and the only-TACE group.

\begin{tabular}{lccc}
\hline & $\begin{array}{c}\text { TACE } \\
(\mathrm{n}=120)\end{array}$ & $\begin{array}{c}\text { TACE+RT } \\
(\mathrm{n}=13)\end{array}$ \\
\hline Age & $68.6 \pm 0.9$ & $75.8 \pm 2.1$ & $p=0.0047$ \\
Sex (male $:$ female) & $77: 43$ & $4: 9$ & $p=0.0191$ \\
Child-Pugh (A : B : C) & $81: 39: 0$ & $13: 0: 0$ & $p=0.0145$ \\
Etiology (HCV : HBV : NBNC : HCV+HBV) & $78: 23: 1: 18$ & $9: 1: 3: 0$ & $p=0.6867$ \\
AFP & $951.0 \pm 407.3$ & $448.2 \pm 326.1$ & $p=0.9601$ \\
AFP-L3 & $16.3 \pm 2.1$ & $15.6 \pm 6.7$ & $p=0.7273$ \\
PIVKA-II & $3,133.55 \pm 1,462.2$ & $25.1 \pm 4.09$ & $p=0.0075$ \\
Tumor size (cm) & $2.8 \pm 0.1$ & $2.5 \pm 0.3$ & $p=0.3278$ \\
Tumor number $(1: 2-3: \geq 4)$ & $38: 34: 48$ & $11: 1: 1$ & $p=0.0008$ \\
TNM staging (I : II : III : IV A) & $10: 53: 5: 6$ & $5: 7: 0: 1$ & $p=0.0015$ \\
CLIP score (0: $: 2: 3: 4: 5)$ & $59: 55: 6: 0: 0$ & $5: 6: 1: 0: 1$ & $p=0.0216$ \\
\hline
\end{tabular}

Data were expressed as mean \pm SE. Age, AFP, AFP-L3, PIVKA-II, Tumor sizes were analyzed by Wilcoxon's test. Other data were analyzed by the $\chi^{2}$ test.

Table 7. Changes in the Child-Pugh score.

\begin{tabular}{|c|c|c|c|}
\hline & $\begin{array}{c}\mathrm{CP} \\
\text { (Before RT) }\end{array}$ & $\begin{array}{c}\mathrm{CP} \\
(6 \text { months after RT) }\end{array}$ & \\
\hline Alternative therapy group & $5.6 \pm 0.5$ & $5.7 \pm 0.7$ & $p=0.2660$ \\
\hline TACE group & $5.3 \pm 0.9$ & $5.8 \pm 1.3$ & $p=0.7287$ \\
\hline
\end{tabular}

Data were expressed as mean $\pm \mathrm{SD}$. Data were analyzed by Wilcoxon's test.

months in the TACE group (Fig. 4A). This duration of time to recurrence was similar to that in the RFA or resection group. Further, we compared the RT (TACE group) and only-TACE patients (Table 6). We chose the only-TACE patients in Fig. 1A. There were 577 TACE patients in our hospital. Because TACE is usually performed for advanced HCC, TACE patients are progressing than patients of RT (+TACE) as substitute for local treatment. Therefore, we selected 120 patients (the first TACE patients) who did not have the treatment history of TACE, because of the expected therapeutic effect. The patients with RT (TACE group) had a significantly longer disease-free survival than the first TACE group (Fig. 4B).

\section{Adverse effect}

Two patients in the Stage IV A group discontinued treatment because of cytopenia and a liver abscess, respectively. However, almost no side effects occurred. The known side effects of RT included acute toxicities and late toxicities. Acute side effects are elevations in liver enzymes, thrombocytopenia, leukopenia, and nausea. In our study, most of these were grade 1 to grade 2 .

Only three patients used granulocyte-colony stimulating factor for neutropenia in the alternative therapy group and the TACE group, and no patients discontinued treatment. We analyzed liver function after 6 months for evaluation of late toxicities (Table 7). We could not evaluate the disease progression for the Stage IV A group. The alterna- tive therapy and TACE group showed no decrease in liver function after 6 months.

\section{Discussion}

In our hospital, the purpose of performing RT could be divided into three groups. In the alternative therapy group and the TACE group, the RT was chosen as a substitute for resection and ablation. In the Stage IV A group, RT was chosen as the treatment for advanced HCC. RT has a potential role in all stages of HCC (Fig. 5). However, RT is not generally considered an option in consensus documents or national guidelines, primarily because of the lack of sufficient evidence supporting its effectiveness.

Many patients in the Stage IV A group presented with vascular invasion, such as portal vein tumor thrombosis (PVTT). In 1989, Takagi et al. reported the irradiation of HCC with PVTT (Takagi et al. 1989). Since then, many studies have reported the effect of RT on HCC with PVTT (Huang et al. 2009; Zhang et al. 2009; Rim et al. 2012). The median survival period is reported to be 4 to 15 months. The median survival period was $9.7 \pm 1.7$ months in our Stage IV A group. In our study, 10 out of 27 patients showed Vp3 or higher involvement. The survival rate was relatively good because half the cases had non-Vp3 involvement. However, the result was excellent because our Stage IV A group already included many patients who were resistant to other treatments such as HAIC and TACE. Our result was considered to be better than observed in the 


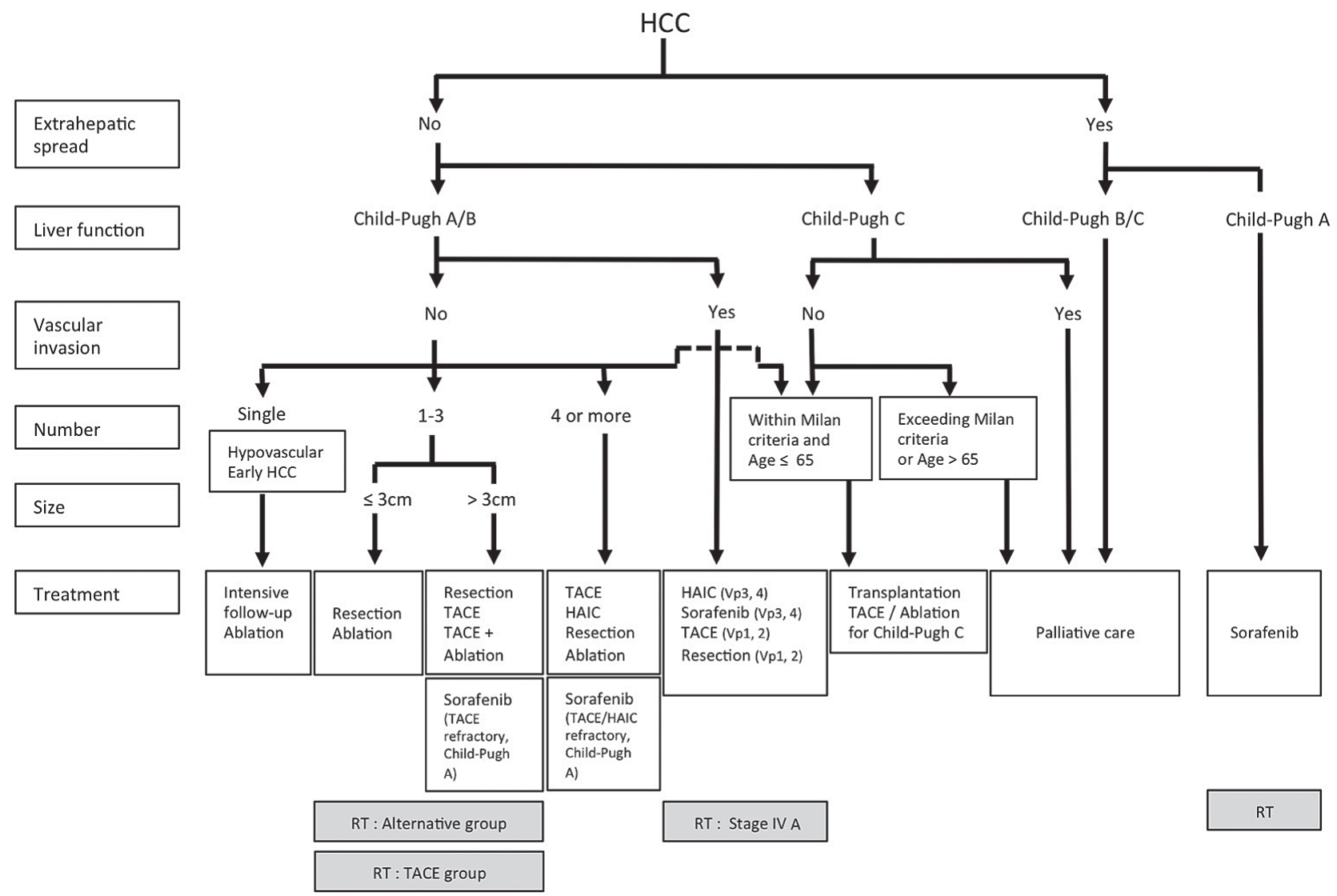

Fig. 5. The algorithm by the Japan Society of Hepatology for positioning for RT therapy.

natural history of $\mathrm{Vp} 3$ patients.

In this study, we also compared the results of radical irradiation and sorafenib. There were no significant differences between the two groups, probably because the number of patients in the sorafenib group was so small. Our result might suggest that RT was at least not inferior to sorafenib. However, the overall survival of our sorafenib group was shorter than that in the SHARP trial (Llovet et al. 2008), possibly because we excluded cases of stage III or stage IVB disease with extrahepatic metastasis. These types of patients are expected to show long-term survival with sorafenib.

HCC presents a high recurrence rate and treatment becomes difficult for various reasons with advancing age. When an elderly patient comes to the hospital, they might already have dementia, which could make hospitalization difficult, and so remaining at home may be preferred by their families. Outpatient treatment could be effective in such cases. We thought that the median survival period after RT of $29.2 \pm 3.6$ months in the alternative therapy group appeared to better than that of patients under no treatment with observation. Because the disease free-survival rate after RT was $13.0 \pm 2.8$ months, they did not show a decrease in liver function at 6 months after RT. It is, therefore, a treatment that warrants further investigation.

To improve the therapeutic effect, RT was added after TACE in the TACE group based on previous reports (Seong et al. 1999; Shim et al. 2005), which showed better outcomes than with TACE alone. Compared to single TACE, these reports showed a good therapeutic result by adding RT after TACE. We compared the effectiveness of TACE with curative treatments such as surgical resection and RFA. The TACE group tended to show early recurrences compared to the patients of the curative treatment group. However, the TACE group did not show any obvious inferiority in disease-free survival. The patients who received RT after TACE showed excellent results, especially given the limited treatments options for HCC. We believe that RT after TACE should be considered for local control of HCC with a high degree of malignancy, such as the simple nodular type with extra-nodular growth, when surgery or RFA would be difficult for some reason.

Unfortunately, the number of cases in our study was small. However, the number of cases is limited because RT is not a treatment mentioned in the guidelines. Because we divided the cases into three groups in this study, the number of cases became even smaller.

Although there were only a few cases, a curative effect was shown for HCC at different stages. RT has been shown to be adaptable to many different stages of HCC (Fig. 5). It could become a substitute treatment for relatively early stages of cancer in TACE or alternative groups. The Stage IV A group, however, had advanced HCC. Chemoradiation therapy (CRT) is often performed for other solid carcino- 
mas and has been shown to be effective. The use of sorafenib in a combination therapy with RT for HCC has been reported (Yu et al. 2013). In addition, new methods such as stereotactic radiotherapy and particle radiotherapy are increasing the effectiveness of RT. Given the consistent effect of RT for HCC, it should be considered in a variety of conditions.

\section{Conflict of Interest}

The authors declare no conflict of interest.

\section{References}

Cheng, A.L., Kang, Y.K., Chen, Z., Tsao, C.J., Qin, S., Kim, J.S., Luo, R., Feng, J., Ye, S., Yang, T.S., Xu, J., Sun, Y., Liang, H., Liu, J., Wang, J., et al. (2009) Efficacy and safety of sorafenib in patients in the Asia-Pacific region with advanced hepatocellular carcinoma: a phase III randomised, double-blind, placebo-controlled trial. Lancet Oncol., 10, 25-34.

Feng, M. \& Ben-Josef, E. (2011) Radiation therapy for hepatocellular carcinoma. Semin. Radiat. Oncol., 21, 271-277.

Huang, Y.J., Hsu, H.C., Wang, C.Y., Wang, C.J., Chen, H.C., Huang, E.Y., Fang, F.M. \& Lu, S.N. (2009) The treatment responses in cases of radiation therapy to portal vein thrombosis in advanced hepatocellular carcinoma. Int. J. Radiat. Oncol. Biol. Phys., 73, 1155-1163.

Kim, M.N., Kim, B.K. \& Han, K.H. (2013) Hepatocellular carcinoma in patients with chronic hepatitis $\mathrm{C}$ virus infection in the Asia-Pacific region. J. Gastroenterol., 48, 681-688.

Klein, J. \& Dawson, L.A. (2013) Hepatocellular carcinoma radiation therapy: review of evidence and future opportunities. Int. J. Radiat. Oncol. Biol. Phys., 87, 22-32.

Komatsu, S., Fukumoto, T., Demizu, Y., Miyawaki, D., Terashima, K., Sasaki, R., Hori, Y., Hishikawa, Y., Ku, Y. \& Murakami, M. (2011) Clinical results and risk factors of proton and carbon ion therapy for hepatocellular carcinoma. Cancer, 117, 4890-4904.

Kudo, M., Izumi, N., Kokudo, N., Matsui, O., Sakamoto, M., Nakashima, O., Kojiro, M. \& Makuuchi, M. (2011) Management of hepatocellular carcinoma in Japan: Consensus-Based Clinical Practice Guidelines proposed by the Japan Society of Hepatology (JSH) 2010 updated version. Dig. Dis., 29, 339-364.

Lau, W.Y. \& Lai, E.C. (2008) Hepatocellular carcinoma: current management and recent advances. Hepatobiliary Pancreat. Dis. Int., 7, 237-257.

Llovet, J.M., Bru, C. \& Bruix, J. (1999) Prognosis of hepatocellular carcinoma: the BCLC staging classification. Semin. Liver Dis., 19, 329-338.

Llovet, J.M., Real, M.I., Montana, X., Planas, R., Coll, S., Aponte, J., Ayuso, C., Sala, M., Muchart, J., Sola, R., Rodes, J. \& Bruix, J. (2002) Arterial embolisation or chemoembolisation versus symptomatic treatment in patients with unresectable hepatocellular carcinoma: a randomised controlled trial. Lancet, 359, 1734-1739.

Llovet, J.M., Ricci, S., Mazzaferro, V., Hilgard, P., Gane, E., Blanc, J.F., de Oliveira, A.C., Santoro, A., Raoul, J.L., Forner, A., Schwartz, M., Porta, C., Zeuzem, S., Bolondi, L., Greten, T.F., et al. (2008) Sorafenib in advanced hepatocellular carcinoma. N. Engl. J. Med., 359, 378-390.

Lo, S.S., Dawson, L.A., Kim, E.Y., Mayr, N.A., Wang, J.Z., Huang, Z. \& Cardenes, H.R. (2010) Stereotactic body radiation therapy for hepatocellular carcinoma. Discov. Med., 9, 404-410.

Lo, C.M., Ngan, H., Tso, W.K., Liu, C.L., Lam, C.M., Poon, R.T., Fan, S.T. \& Wong, J. (2002) Randomized controlled trial of transarterial lipiodol chemoembolization for unresectable hepatocellular carcinoma. Hepatology, 35, 1164-1171.

Mittal, S. \& El-Serag, H.B. (2013) Epidemiology of hepatocellular carcinoma: consider the population. J. Clin. Gastroenterol., 47 Suppl, S2-6.

Rim, C.H., Yang, D.S., Park, Y.J., Yoon, W.S., Lee, J.A. \& Kim, C.Y. (2012) Effectiveness of high-dose three-dimensional conformal radiotherapy in hepatocellular carcinoma with portal vein thrombosis. Jpn. J. Clin. Oncol., 42, 721-729.

Seong, J., Keum, K.C., Han, K.H., Lee, D.Y., Lee, J.T., Chon, C.Y., Moon, Y.M., Suh, C.O. \& Kim, G.E. (1999) Combined transcatheter arterial chemoembolization and local radiotherapy of unresectable hepatocellular carcinoma. Int. J. Radiat. Oncol. Biol. Phys., 43, 393-397.

Shim, S.J., Seong, J., Han, K.H., Chon, C.Y., Suh, C.O. \& Lee, J.T. (2005) Local radiotherapy as a complement to incomplete transcatheter arterial chemoembolization in locally advanced hepatocellular carcinoma. Liver Int., 25, 1189-1196.

Takagi, H., Takayama, H., Yamada, S., Uehara, M., Ojima, T., Saitoh, S., Katakai, S., Yamada, T., Abe, T., Sakurai, S., Takezawa, J., Nagamine, T. \& Kobayashi, S. (1989) Radiation therapy of hepatocellular carcinoma. Nihon Shokakibyo Gakkai Zasshi, 86, 237-245.

Therasse, P., Arbuck, S.G., Eisenhauer, E.A., Wanders, J., Kaplan, R.S., Rubinstein, L., Verweij, J., Van Glabbeke, M., van Oosterom, A.T., Christian, M.C. \& Gwyther, S.G. (2000) New guidelines to evaluate the response to treatment in solid tumors. European Organization for Research and Treatment of Cancer, National Cancer Institute of the United States, National Cancer Institute of Canada. J. Natl. Cancer Inst., 92, 205-216.

Yu, W., Gu, K., Yu, Z., Yuan, D., He, M., Ma, N., Lai, S., Zhao, J., Ren, Z., Zhang, X., Shao, C. \& Jiang, G.L. (2013) Sorafenib potentiates irradiation effect in hepatocellular carcinoma in vitro and in vivo. Cancer Lett., 329, 109-117.

Zhang, X.B., Wang, J.H., Yan, Z.P., Qian, S., Du, S.S. \& Zeng, Z.C. (2009) Hepatocellular carcinoma with main portal vein tumor thrombus: treatment with 3-dimensional conformal radiotherapy after portal vein stenting and transarterial chemoembolization. Cancer, 115, 1245-1252. 